\title{
Eye Drops, Suspension in Single-dose Container
}

National Cancer Institute

\section{Source}

National Cancer Institute. Eye Drops, Suspension in Single-dose Container. NCI

Thesaurus. Code C149509.

Medicinal product consisting of an eye drops suspension presented in a single-dose container. 